\title{
In situ bulge testing in an atomic force microscope: Microdeformation experiments of thin film membranes
}

\author{
E.W. Schweitzer ${ }^{\text {a) }}$ and M. Göken \\ University Erlangen-Nürnberg, Department of Materials Science and Engineering, Institute I, \\ D-91058 Erlangen, Germany
}

(Received 11 April 2007; accepted 28 June 2007)

\begin{abstract}
A new bulge testing setup for the measurement of the mechanical properties of thin films is presented. This self-built device can be incorporated in an atomic force microscope (AFM), which allows the recording of topographic images of the observed sample membranes under load conditions. Bulge test experiments on different silicon nitride films are presented and compared to nanoindentation experiments. The measured elastic moduli from nanoindentation and bulge testing are in good agreement. Apart from that, the ability to extract stress-strain data from AFM scans is shown, and the results are compared to standard bulge testing experiments. Imaging of the sample microstructure under load conditions is demonstrated on a thin $\mathrm{Cu}$ film.
\end{abstract}

\section{INTRODUCTION}

Due to the increasing application of microelectromechanical devices (MEMS) in recent years, the mechanical properties of thin films have become an important research topic. Basically there are two major reasons why the thin-film mechanical behavior is of interest: the technical reason, i.e., the material properties (e.g., Young's modulus and residual stress of thin films are very much dependent on the manufacturing conditions; and the scientific reason, i.e., with decreasing film thickness, the mechanical properties deviate from that of bulk materials because the sample dimensions are in an order of magnitude where physical effects, such as dislocation motion, occur. ${ }^{1}$ Beams and co-workers ${ }^{2}$ introduced bulge testing of thin freestanding sample membranes in the 1950 s as a way to quantify their stress-strain behavior without suffering from substrate influences. The principle of bulge testing is to record the load-deflection $(p-h)$ data of a freestanding membrane, which is uniformly pressurized from one side. Assuming that the bending stiffness is negligible (which is the case as long as the membrane thickness $t$ is four orders of magnitude smaller than the membrane width $2 a$ ), load deflection follows the equation:

$$
p=\frac{C_{1} \sigma_{0} t}{a^{2}} h+\frac{C_{2} E t}{a^{4}} h^{3},
$$

where $\sigma_{0}$ and $E$ denote residual stress and Young's modulus, respectively. $C_{1}$ and $C_{2}$ are constants that de-

\footnotetext{
a) Address all correspondence to this author. e-mail: elmar.schweitzer@ww.uni-erlangen.de DOI: $10.1557 / J M R .2007 .0373$
}

pend on the aspect ratio of the membrane. $C_{2}$ is also slightly dependent on Poisson's ratio v. ${ }^{3}$

The stress state in the membrane can be trimmed by variation of the membrane shape. Early experiments were done on circular membranes, but as soon as silicon micromachining processes were available, square and rectangular membranes became common. The stress state in the center of such a square film is biaxial, with $C_{1}=$ 3.393 and $C_{2}=1 /[(1-v)(0.8+0.062 v)] .^{4}$ For rectangular membranes having an aspect ratio of $b / a \geqslant 4$, the constants become independent of membrane shape: $C_{1}=2 ; C_{2}=4 / 3\left(1-v^{2}\right) .{ }^{5}$ A detailed derivation of Eq. (1) can be found elsewhere. ${ }^{6}$

In this special case of rectangular membranes, the strain in the $y$ direction can be neglected and using Hooke's law the strain $\epsilon_{x}$ is given by

$$
\begin{gathered}
\epsilon_{y}=\frac{1}{E}\left(\sigma_{y}-\nu \sigma_{x}\right) \equiv 0, \sigma_{y}=v \sigma_{x}, \\
\epsilon_{x}=\frac{1}{E}\left(\sigma_{x}-\nu \sigma_{y}\right)=\sigma_{x} \frac{1-v^{2}}{E} .
\end{gathered}
$$

The definition of the $x, y$, and $z$ directions can be seen in Fig. 1. This state is referred to as the plane-strain condition, and $M=E /\left(1-v^{2}\right)$ is called the plain-strain modulus. For such membranes, the $p-h$ data transfer directly to stress and strain $(h \ll a)^{16}$

$$
\begin{aligned}
\sigma_{x} & =\frac{p a^{2}}{2 h t}, \\
\epsilon_{x} & =\frac{2 h^{2}}{3 a^{2}} .
\end{aligned}
$$

A derivation can be found in Appendix A. 


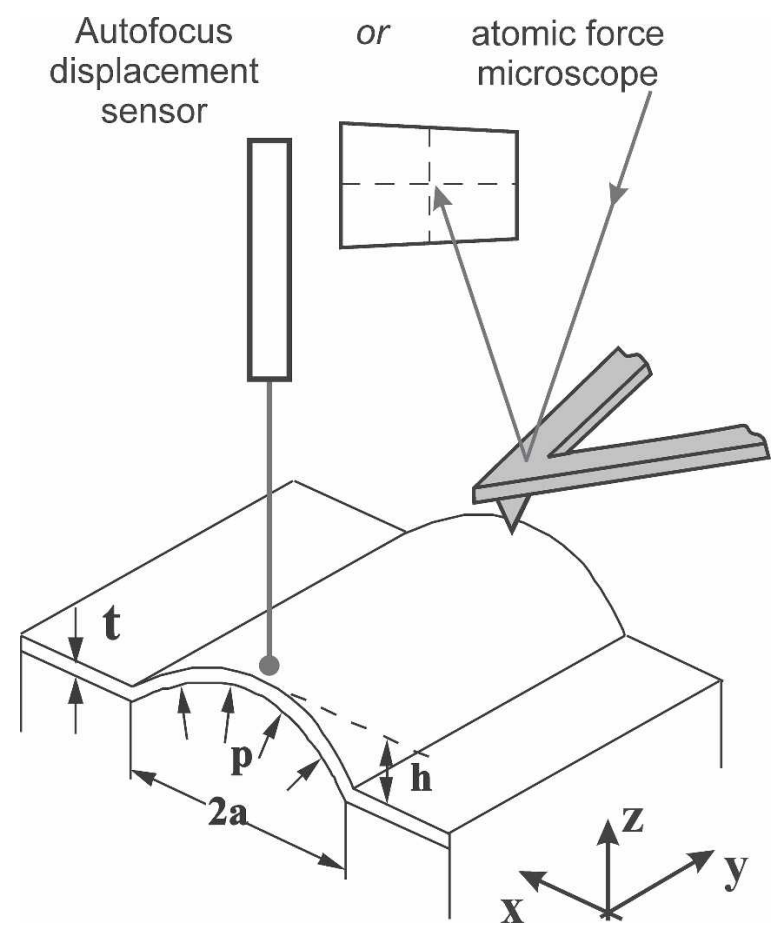

FIG. 1. Possibilities of displacement detection: laser autofocus displacement sensor for the recording of pressure-displacement curves or AFM for in situ bulge testing and membrane curvature analysis.

In a normal stress-strain plot, the residual stress $\sigma_{0}=$ $\left.\sigma_{x}\right|_{\epsilon_{x}=0}$ can be determined from the ordinate offset, which makes bulge testing a tool to study the influence of the deposition parameters on the residual stresses of thin films.

This method, however, provides integral information of the mechanical properties and is therefore comparable to tensile testing. The combination of a bulge test device with an atomic force microscope (AFM) enables the investigation of effects on a local, microstructural level (Fig. 1). An AFM is a useful tool to record sample topography with nanometer resolution in lateral directions and even subnanometer resolution in normal direction. ${ }^{6}$ A sharp tip at the end of a flexible cantilever is scanned across the surface. The translation in the surface plane as well as in normal direction is accomplished by a piezoscanner. In this paper, the first bulge test experiments performed under an AFM cantilever tip are described.

\section{EXPERIMENTAL}

Traditionally bulge testing is done, e.g., with a motordriven syringe pump to increase oil or water pressure beneath the membrane and the deflection is measured interferometrically. ${ }^{7,8}$ The new setup, described in this paper, is the first bulge tester, which can be used in an AFM to perform in situ imaging of the membrane under load-conditions. Instead of using liquids to pressurize the membranes, a gas pressure system is used, which is more flexible in loading and unloading the membrane.

\section{A. Pressure control}

Loading of the membrane in the bulge tester is done by applying a gas pressure to the membrane dynamically, i.e., a constant $\mathrm{N}_{2}$ flow is established in the system and the difference between the pressure beneath the sample and the ambient pressure is adjusted by controlling the outlet valve. A similar setup has been reported in Ref. 9. The pressure difference is detected by a pressure transducer which is thermostatically kept constant at $45^{\circ} \mathrm{C}$ to minimize thermal drift and a pressure resolution of $240 \mathrm{~Pa}$ at $240 \mathrm{kPa}$ maximum detectable pressure is achieved with this system (Fig. 2). The advantage of such a setup compared to, e.g., a motor-driven syringe pump is its higher flexibility. The pressure can be increased or decreased at arbitrary rates, limited just by the capability of the feedback control system.

\section{B. Bulge height detection}

The newly developed bulge tester offers two different possibilities to measure the bulge height: measurement by an additional displacement detector and measurement of the curvature of the membrane with an AFM. The best resolution is achieved with an additional displacement detector as described herein. The bulge height is recorded with a laser auto focus displacement sensor working on the principle of dynamic focusing: The light of a laser diode is brought into a parallel beam and then guided in such a way that $10 \mathrm{~mm}$ below the outlet a focal point with a radius of $\sim 2 \mu \mathrm{m}$ is formed on the membrane surface. The light reflected from the surface is assessed by the objective and directed to a focus detector. The detector converts the optical signal into an electrical one which contains information on the distance from the optical

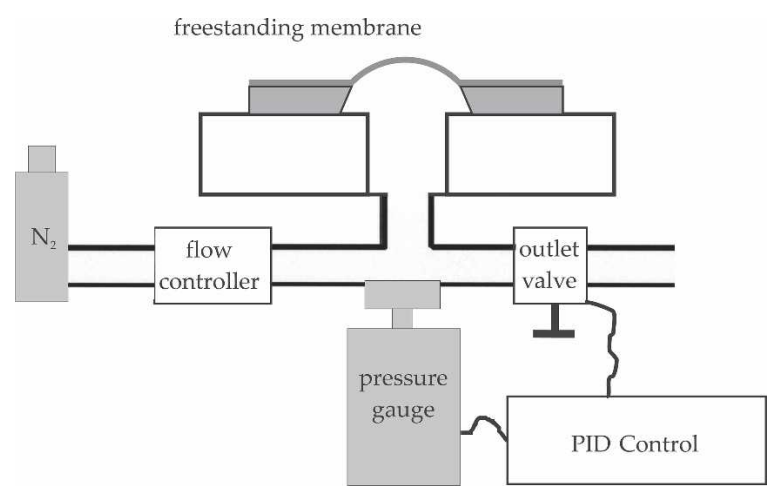

FIG. 2. Sketch of the bulge test's pressure control setup. The gas pressure inside the system is measured (pressure gauge), and the outlet valve is feedback-controlled (PID). Data aquisition is done with a commercial PC. The flow controller ensures a constant gas flow beneath the sample, which is independent of the pressure inside the system. 
system to the surface. A linear motor moves the objective to keep the laser beam in focus throughout the test routine. The detector covers a measuring range of $600 \mu \mathrm{m}$ having a resolution of $<200 \mathrm{~nm}$. In a calibration test against a strain gauge-monitored piezoactuator, a sinusoidal displacement amplitude of $90 \mathrm{~nm}$ could be detected. The resolution is slightly dependent on the surface reflectivity. On very smooth and shiny surfaces as well as on partially transparent samples, finding the focal point is getting more difficult, resulting in a lower signal-to-noise ratio. A detailed discussion of alignment issues can be found in Appendix B.

The base plate can substitute the standard sample mount of a Dimension 3100 AFM (Digital InstrumentsVeeco Metrology Group, Chadds Ford, PA). The piezoscanner of the AFM can be replaced by the laser autofocus sensor. In this mode of operation, the AFM simply serves as a lateral and vertical positioning system. Substituting the displacement sensor with the AFM scanner allows topographic imaging of the film surface (Fig. 1). Data collection as well as pressure control is realized by a LabView (National Instruments, Austin, TX) application running on a standard personal computer and a hardware PID-Controller (MKS, Andover, MA).

\section{Sample preparation}

Four different silicon nitride sample films have been deposited on $\langle 100\rangle$ silicon wafers to serve as a model material (Table I): low-pressure chemical vapor deposited (LPCVD) nitride with a thickness of $100 \mathrm{~nm}$ (type 1), stress-optimized LPCVD nitride with a thickness of $450 \mathrm{~nm}$ (type 2), plasma-enhanced chemical vapor deposited (PECVD) nitride with a thickness of $450 \mathrm{~nm}$ (type 3), and a bilayer consisting of 100-nm LPCVD nitride on top of 50-nm thermally grown $\mathrm{SiO}_{2}$ (type 4). LPCVD nitride in general bears very high tensile residual stresses resulting in rather high interfacial forces which increase with increasing film thickness. Therefore the oxide sublayer of sample film type 4 serves as a stress buffer between the silicon substrate and the highly stressed nitride layer because the inward diffusion of oxygen into Si during the oxidation process leads to com-

TABLE I. Type of nitride films studied with corresponding film thickness $t$, measured Young's modulus $E$, and residual stress $\sigma_{\mathrm{o}}$.

\begin{tabular}{|c|c|c|c|c|}
\hline Number & $\begin{array}{l}\text { Film } \\
\text { thickness } \\
t(\mathrm{~nm})\end{array}$ & Type of film & $\begin{array}{c}E \\
(\mathrm{GPa})\end{array}$ & $\begin{array}{c}\sigma_{\mathrm{o}} \\
(\mathrm{MPa})\end{array}$ \\
\hline 1 & 100 & $\operatorname{LPCVD~SiN}_{x}$ & 295.4 & 1068 \\
\hline 2 & 450 & Stress optimized LPCVD $\mathrm{SiN}_{x}$ & $\mathrm{n} / \mathrm{a}$ & $\mathrm{n} / \mathrm{a}$ \\
\hline 3 & 450 & $\operatorname{PECVD~SiN}_{\mathrm{x}}$ & 92.1 & $\approx 10$ \\
\hline 4 & $100 / 50$ & $\begin{array}{l}\mathrm{LPCVD} \mathrm{SiN}_{x} / \text { thermally grown } \\
\mathrm{SiO}_{2} \text { (double layer) }\end{array}$ & 176.6 & 712 \\
\hline 5 & $104 / 64$ & $\begin{array}{l}\mathrm{LPCVD} \mathrm{SiN}_{x} \text { /thermally grown } \\
\mathrm{SiO}_{2} \text { (double layer) }\end{array}$ & 107.9 & 525 \\
\hline
\end{tabular}

pressive stresses in the $\mathrm{SiO}_{2}$. The topography of all films studied is shown in Fig. 3. The high roughness of the LPCVD nitride films compared to the others is obvious.

The nitride on the wafer backside has been patterned using standard photolithography followed by subsequent dry etching. This leads to the exposure of the Si substrate in the dry-etched regions. The openings have the desired membrane shape. Freestanding membranes have been produced by anisotropic wet etching of the Si-substrates at $50{ }^{\circ} \mathrm{C}$ in a potassium hydroxide $(\mathrm{KOH})$ solution. The silicon nitride is not attacked by the solution, whereas $\mathrm{Si}$ dissolves slowly in $\mathrm{KOH}$. The $\langle 100\rangle$ planes etch about two orders of magnitude faster than the $\langle 111\rangle$ planes resulting in perfectly rectangular orifices with $\langle 111\rangle$ side walls and the nitride membrane remaining on the wafer front side. All membranes had an aspect ratio of $b / a>4$ with different values of $a$. The windows have been epoxy-glued on a sample holder that can easily be screwed onto the machine's pressure chamber.

Compressive residual stresses have to be avoided in preparation of membranes because they lead to a buckling behavior. For example membranes of type 2 showed such buckling, as can be seen in Fig. 5. Consequently, these membranes could not be bulge tested. Catlin and Walker ${ }^{10}$ tried to solve the problem of slack circular membranes by applying an infinitesimal pressure until the membrane does not show any wrinkles anymore and modified Eq. (5) to $\epsilon_{x}=2\left(h^{2}-h_{0}{ }^{2}\right) / 3 a^{2}, h_{0}$ being the initial bulge height at this point. However, Small and $\mathrm{Nix}^{11}$ carefully examined the influence of slacking on the stress distribution within the membrane in a finite element analysis. Due to the clamping of the film at the membrane edge, the circumferential stress at the edge stays compressive even though the radial stress in the center of the membrane is highly tensile. There is no model that accounts for such complicating stress states. Furthermore, compressive stresses in rectangular membranes lead to "phone cord-like" wrinkles (Fig. 4), which show discontinuities in the $p-h$ curve when pressurized, which makes an analysis of bulge test data impossible.

\section{Nanoindentation}

Despite the fact that instrumented indentation experiments suffer from substrate influence, ${ }^{12}$ when the indented sample film gets thinner and thinner, nanoindentation became a standard tool in thin-film characterization. ${ }^{13}$ Nanoindentation experiments have been used to get reference values for the elastic stiffness of the sample films, which can be compared to the bulge test results. The measurements have been performed in a nanoindenting atomic force microscope (NI-AFM; Hysitron TriboScope system, Hysitron, Inc., Minneapolis, MN). In this system, a three-sided pyramidal diamond indenter is used to both scan the surface topography and perform indentations. The indents were done on the sample film on $\mathrm{Si}$ 


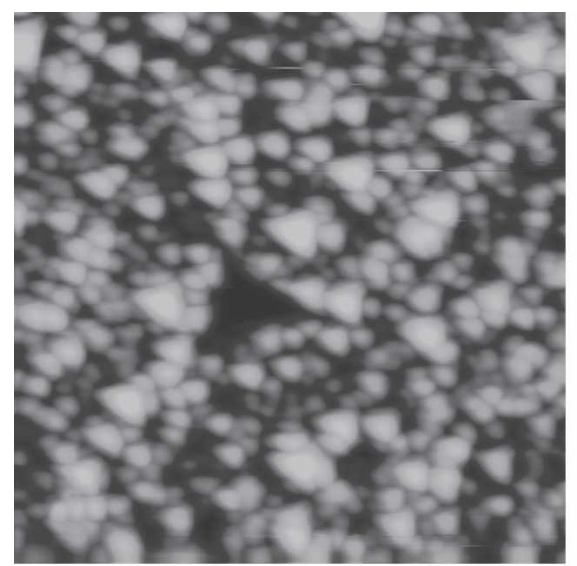

(a)

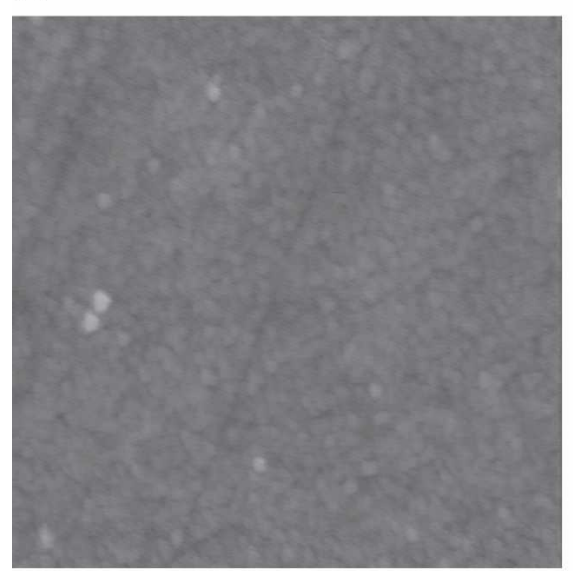

(c)

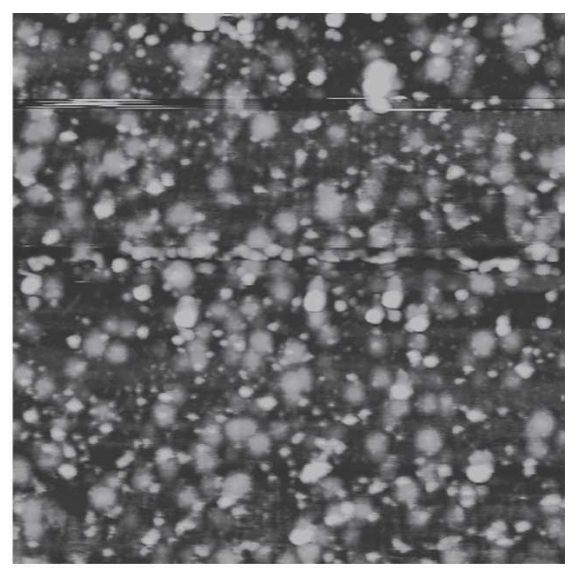

(b)

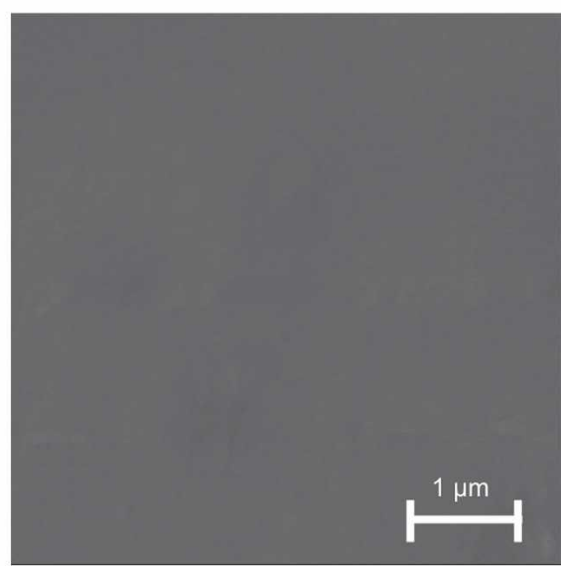

(d)

FIG. 3. Topographic AFM scan of the studied films: (a) 100-nm LPCVD nitride (type 1), (b) 450-nm LPCVD nitride (type 2), (c) 450-nm PECVD nitride (type 3), and (d) 100-nm LPCVD nitride on 50-nm $\mathrm{SiO}_{2}$ (type 4); the scale applies to all images.

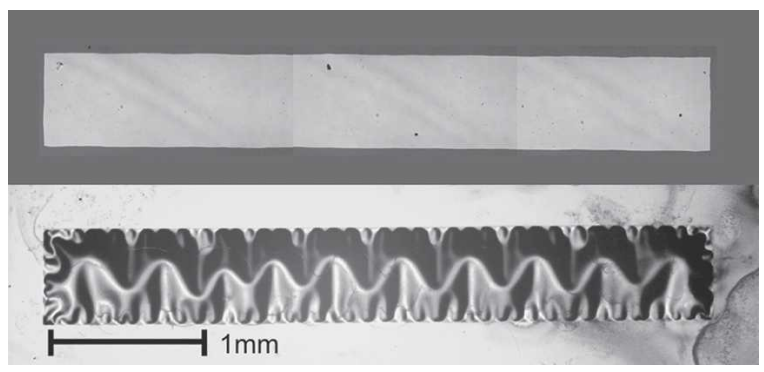

FIG. 4. Comparison of a flat membrane of type 3 (top) and a buckled membrane with "phone cord-like" wrinkles of type 2 (below).

substrate. To facilitate the comparison of nanoindentation experiments of films with different thicknesses, the relative indentation depth $h_{\max } / t$ (tip displacement into surface at maximum load over film thickness) is introduced, i.e., the position of the interface between film and substrate corresponds to $h_{\max } / t=1$ (Fig. 5). Loaddisplacement $(P-h)$ curves are taken during the indentation process and hardness $H$ and reduced Young's modulus $E_{\mathrm{r}}$ are determined by the so-called Oliver-Pharr method. ${ }^{14}$

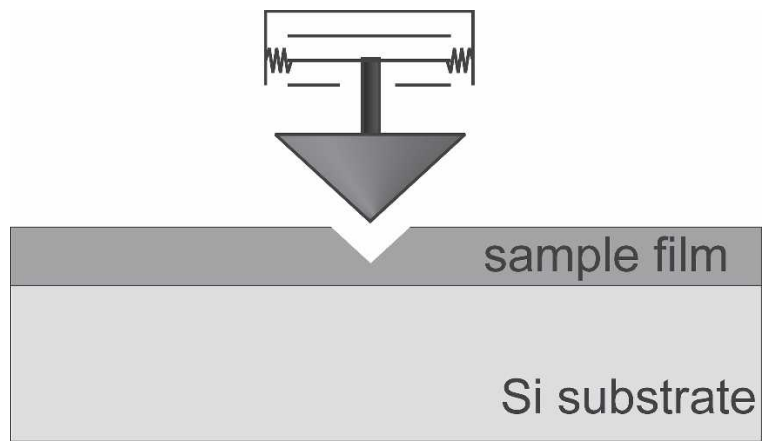

FIG. 5. Pyramidal indenter is loaded by an electrostatic force transducer and the displacement of the tip as a function of load is recorded. The relative indentation depth $h_{\max } / t$ facilitates the comparison data acquired from sample films of different thickness, e.g., the depicted indent corresponds to $h_{\max } / t \approx 0.6$.

The reduced modulus accounts for the elastic deformation of the diamond tip during indentation and is defined as

$$
\frac{1}{E_{\mathrm{r}}}=\frac{1-v_{\text {sample }}^{2}}{E_{\text {sample }}}+\frac{1+v_{\text {tip }}^{2}}{E_{\text {tip }}} .
$$


The parameters of the diamond tip are $1141 \mathrm{GPa}$ and 0.07 for $E_{\text {tip }}$ and $v_{\text {tip }}$, respectively. All values of Young's modulus presented in this paper are deduced from $E_{\mathrm{r}}$. According to Ref. 15, the Poisson's ratio if $\mathrm{Si}_{3} \mathrm{~N}_{4}$ lies between 0.3 for dense nitride and 0.23 for nitride having a porosity of $20 \%$. Therefore, Poisson's ratio of 0.25 for all silicon nitride films studies is assumed.

Unlike $P-h$ curves of metallic specimens, the timedependent deformation at maximum load is negligible on silicon nitride. Therefore it is justified to use a loadprofile without any hold segment, which allows the contact stiffness to be determined for increasing indentation depth in just one indent by repeated unloading from increasing load levels. The $P-h$ curve of such a profile with 15 unloading branches can be seen in Fig. 6.

\section{RESULTS AND DISCUSSION}

\section{A. Bulge testing}

The first bulge testing results obtained with the displacement sensor are described, where the pressure $p$ is steadily increased. However, different unloading and repeated loading segments can be used, as shown in Fig. 7(a). The hold and unload segments are useful to check whether time-dependent or inelastic deformation is taking place. A typical load-displacement curve is shown in Fig. 7(b). The unloading segments perfectly match with the previous loading segments, showing pure elastic behavior as expected for such silicon nitride films. The plotted data is already corrected for the machine compliance, which can easily be determined by measuring the deflection of the membrane edge as a function of pressure after the actual experiment. The machine compliance depends mainly on the size and the thickness of the wafer substrate. The determined values are summarized in Table II.

The corresponding stress-strain curves as calculated

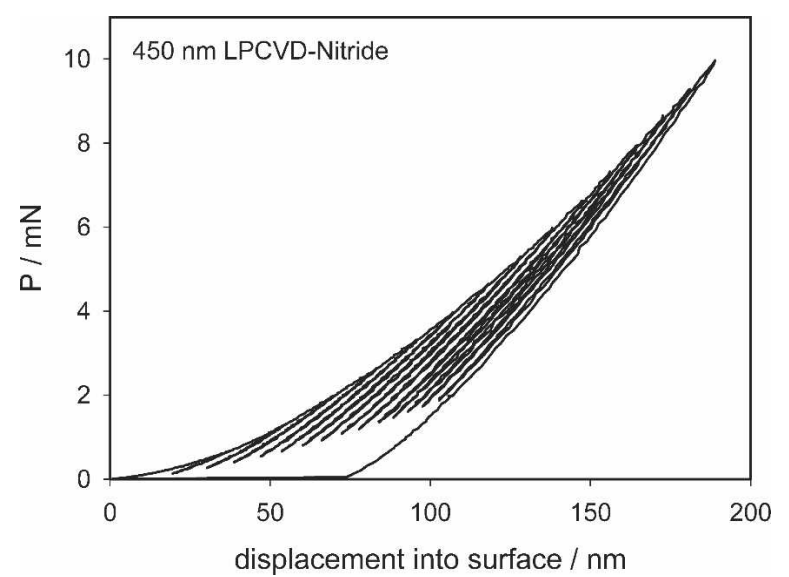

FIG. 6. Load-displacement curve of a nanoindentation experiment for LPCVD nitride film on a silicon substrate with 15 unloading branches.

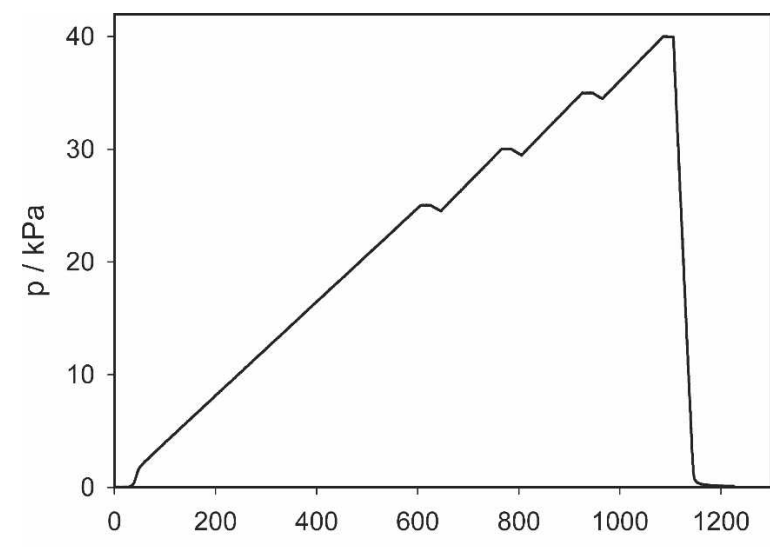

(a)

time / s

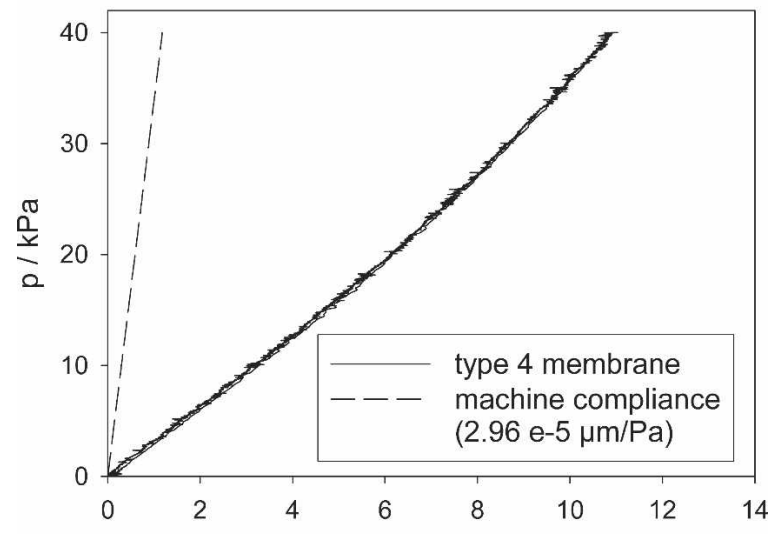

(b)

$\mathrm{h} / \mu \mathrm{m}$

FIG. 7. (a) Example of a typical pressure profile, $p=f(t)$, for bulge testing. (b) Typical load-displacement curve (solid line) and the corresponding machine compliance (dashed line).

TABLE II. Machine compliance for the used wafer thicknesses and membrane shapes.

\begin{tabular}{cccc}
\hline \hline $\begin{array}{c}\text { Wafer } \\
\text { thickness } \\
(\mu \mathrm{m})\end{array}$ & $\begin{array}{c}\text { Membrane } \\
\text { half width } \\
a=255 \mu \mathrm{m}\end{array}$ & $\begin{array}{c}\text { Membrane } \\
\text { half width } \\
a=500 \mu \mathrm{m}\end{array}$ & $\begin{array}{c}\text { Membrane } \\
\text { half width } \\
==1500 \mu \mathrm{m}\end{array}$ \\
\hline 300 & $\ldots$ & $2.96 \times 10^{-5} \mu \mathrm{m} / \mathrm{Pa}$ & $6.19 \times 10^{-4} \mu \mathrm{m} / \mathrm{Pa}$ \\
500 & $1.40 \times 10^{-4} \mu \mathrm{m} / \mathrm{Pa}$ & $\cdots$ & $\cdots$ \\
\hline \hline
\end{tabular}

by Eqs. (4) and (5) for all membranes are plotted in Fig. 8 . The type 4 membrane has been loaded to the maximum load ( $p=100 \mathrm{kPa}$, corresponding to a stress of 1574.5 GPa), all other samples have been loaded until fracture occurred. Young's moduli $E$ have been calculated from the biaxial modulus $M$ assuming a Poisson's ratio of 0.25 . Comparing the LPCVD and the plasmaenhanced chemical vapor deposition (PECVD) nitrides, a significant influence of the production process on both stiffness and residual stress is found. Young's moduli differ by a factor of $>3$. The LPCVD shows a residual stress level of $\sim 1 \mathrm{GPa}$, whereas the PECVD nitride is almost free of stress (Table I). 


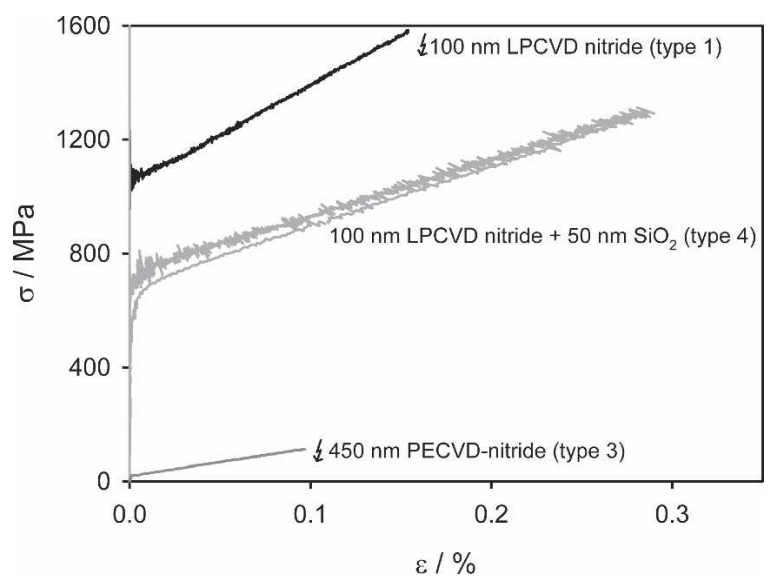

FIG. 8. Stress-strain curves of three types of nitride membranes; thunderbolts mark experiments that ended in film fracture. The unloading branch of the type 4 measurement lies slightly below the loading branch. The time used to increase the pressure was 60 times longer compared to the time the pressure decreased (as can be seen in Fig. 7(a).

Nanoindentation measurements have been performed on all films to receive reference values for the bulge test results. Since nanoindentation measurements of film samples are depth dependent, the results are shown as a function of the relative indentation depth (Fig. 9). The presented values are averaged over five individual indents; the error bars correspond to the standard deviation. The deeper the indenter penetrates the surface, the more substrate influence is noticeable. The measured modulus decreases and approaches the modulus of the substrate. Young's modulus is expected to converge to the bulge test results for decreasing indentation depth, which is the case for the membranes of type 1 and 4 . Jung et al. ${ }^{16}$ compared PECVD and LPCVD silicon nitride films in nanoindentation experiments, plotted Young's moduli as

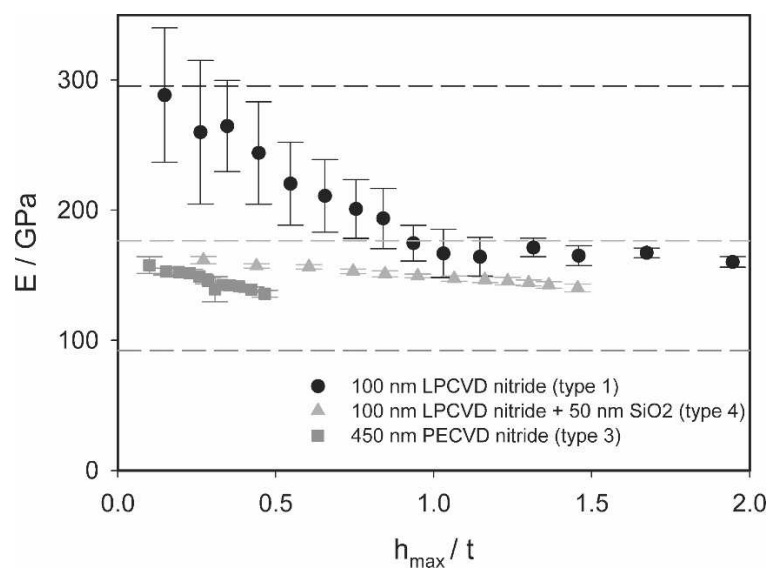

FIG. 9. Comparison of Young's modulus measured with bulge test and nanoindentation; nanoindentation results are shown as a function of relative indentation depth. Dashed lines represent the bulge test results. a function of relative indenter penetration and applied a simple model to extract the film stiffness. The moduli of the films $E_{\mathrm{f}}$ were determined to be $266.4 \mathrm{GPa}$ for LPCVD nitride and 119.3 GPa for PECVD nitride, thus confirming our bulge test results.

Only the nanoindentation measurements of the type 3 membrane deviate significantly from the bulge test values. Both bulge testing and indentation have been repeated and no error could be detected. A possible reason for this discrepancy is, that PECVD silicon nitride is said to be less dense and thus containing a higher defect density than LPCVD nitride. ${ }^{17}$ This may result in different material stiffnesses if one compares compressive loading (like in nanoindentation) to tensile loading (like in bulge testing).

\section{B. Bulge testing in the AFM}

When incorporated in the AFM, the bulge test device can be used in two different ways. First of all, by recording rather large scan areas, the radius of curvature of the bulge $R$ can be determined which can easily be used to compute the bulge height $h$. In contrast to standard bulge testing the curvature analysis gives absolute values of $h$ instead of relative values related to a reference point.

Topographic scans on a bilayer consisting of 104-nm LPCVD nitride on 64-nm $\mathrm{SiO}_{2}$ (type 5 film with a membrane diameter of $2 a=1016 \mu \mathrm{m}$ ) were obtained at different gas pressures $p$. The scanned surface area was a rectangle of $80 \mu \mathrm{m} \times 2.5 \mu \mathrm{m}$ with the longer edge aligned in the $x$ direction (Fig. 10). Polynomial leastsquare fits of second order have been used from which

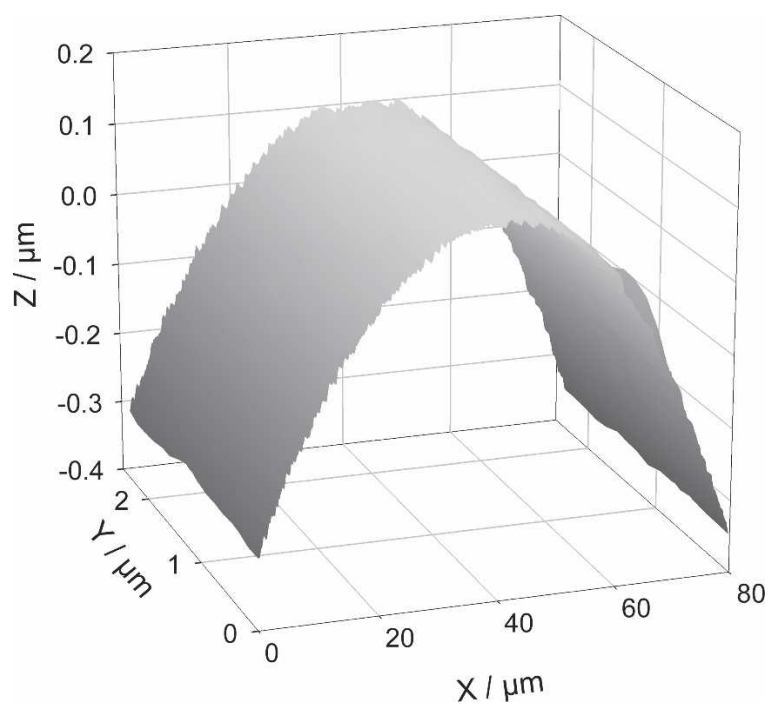

FIG. 10. Surface profile of type 5 membrane (104-nm $\mathrm{Si}_{3} \mathrm{~N}_{4}$ on $64-\mathrm{nm}$ $\mathrm{SiO}_{2}$ ) scanned with an AFM at an applied pressure of $40 \mathrm{kPa}$. 


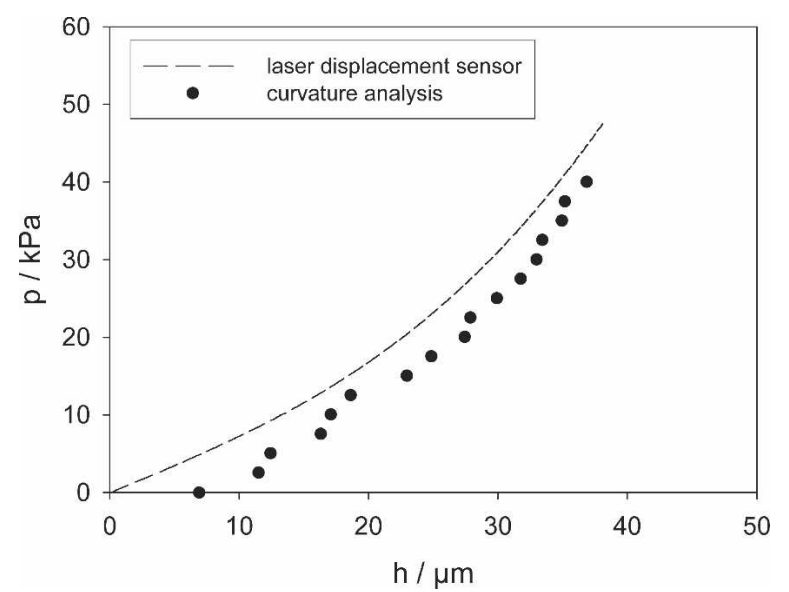

FIG. 11. Pressure-displacement curve as deduced from AFM curvature analysis (dots) compared to laser displacement measurements.

the corresponding radius of curvature $R$ has been calculated by

$$
R=\frac{\left[1+h^{\prime}(x)^{2}\right]^{3 / 2}}{h^{\prime \prime}(x)}
$$

For each scan, a mean radius of curvature $\bar{R}(p)$ has been determined and the bulge height $h$ has been calculated using the Pythagorean theorem $(a \gg h)$ :

$$
h=\frac{a^{2}}{2 R} \text {. }
$$

Figure 11 compares the $p-h$ data measured with the laser displacement sensor to those derived from curvature measurements on the same membrane. An initial bulge height even for $p=0 \mathrm{~Pa}$ can be seen. The bowing error is, according to the AFM-manufacturer, in the order of $50 \mathrm{~nm}$ for a scan area of $90 \mu \mathrm{m},{ }^{18}$ which results in an additional $\Delta h$ of $2.8 \mu \mathrm{m}$ for this specific membrane size $(a=508 \mu \mathrm{m})$. Therefore, the bowing is not the primary reason for this error. Predominantly nonlinear piezo ef- fects, like piezo creep or hysteresis, are responsible for the inaccuracy. The error in $h$ leads to an underestimation of stress and an overestimation of strain. Nevertheless, the stress-strain behavior of the film is qualitatively reproducible.

\section{Microstructural investigations}

The motivation for in situ deformation experiments in an AFM or any other microscope is to observe phenomena on the microstructural scale. Therefore, this AFM-based bulge tester has been developed. In this socalled BisA (Bulge testing in situ in an AFM) mode, the sample surface can be imaged while the film is kept under constant pressure.

To demonstrate the setup's capability to do microstructure imaging under load conditions, a $450-\mathrm{nm} \mathrm{Cu}$ film was electron-beam evaporated on top of a type 4 membrane. The film was heat-treated for $2 \mathrm{~h}$ at $\sim 300{ }^{\circ} \mathrm{C}$ to stabilize the microstructure

Evaporated metal films show a topography, which is caused by the growth process. The as-deposited surface in the membrane's center was "locally polished" by scratching with an scanning tunneling microscope tip. The difference in topography between the as-deposited and the scratched region is clearly visible in Fig. 12. All growth structures were leveled in the modified region on the right-hand side. A detailed view of the modified region is included on the right-hand side, where a grain contrast is visible. The film was in situ pressurized with $80 \mathrm{kPa}$ during scanning, which shows that recording of very fine topographies $(25 \mathrm{~nm}$ height contrast in this case) under pressure is feasible. This is a starting point for further experimental work, like taking a closer look on what is happening on the microstructural level during thin film deformation. This method adds the possibility of in situ imaging of the microstructure to the bulge test's convenient sample handling.

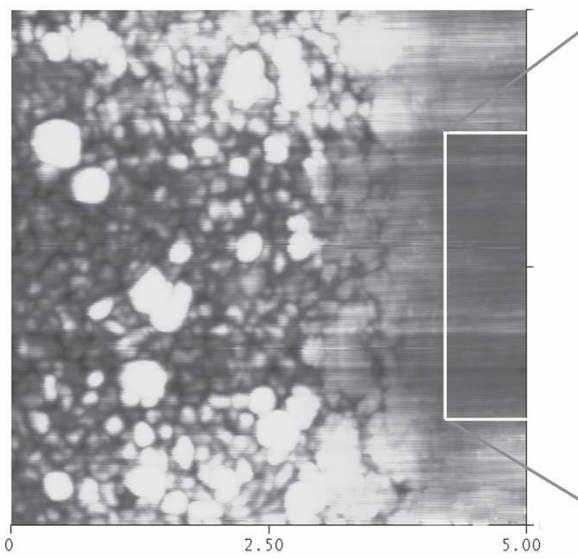

(a)

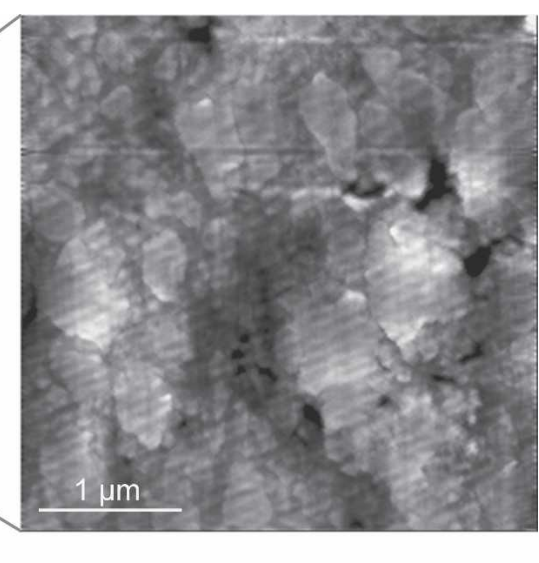

(b)

FIG. 12. (a) AFM micrograph of the as-deposited surface and (b) the locally polished surface of a Cu film on a type 3 membrane imaged at an applied pressure of $80 \mathrm{kPa}$. Note the different height scales of (a) $0-150 \mathrm{~nm}$ and (b) $0-25 \mathrm{~nm}$. 


\section{CONCLUDING REMARKS}

A new bulge test apparatus has been described, which allows in situ AFM imaging under load conditions as well as standard bulge testing. The possibilities of this new bulge test setup have been exemplified by measurements on different silicon nitride thin films. The determined values of Young's modulus are comparable to literature values. Standard bulge testing, where the bulge height is measured with a laser autofocus displacement sensor, and bulge testing in an AFM have been compared. Stress-strain analyses of both methods produce similar data. The system's ability to record topography scans of the samples microstructure under load conditions has been demonstrated and gives rise to further experimental studies.

\section{ACKNOWLEDGMENT}

Christoph Richter of NanoWorld Services GmbH, Erlangen, Germany, is gratefully acknowledged for his advice and the production of the silicon nitride etch-stop layers.

\section{REFERENCES}

1. E. Arzt: Size effects in materials due to microstructural and dimensional constraints: A comparative review. Acta Mater. 46, 5611 (1998).

2. J.W. Beams: Mechanical properties of thin films of gold and silver. In Proceedings, International Conference on Structure and Properties of Thin Films, Bolton Landing, NY, 1959, edited by C.A. Neugebauer, J.B. Newkirk, and D.A. Vermilya (John Wiley, New York, 1959), pp. 183-192.

3. O. Tabata, K. Kawahata, S. Sugiyama, and I. Igarashi: Mechanical property measurements of thin films using load-deflection of composite rectangular membranes. Sens. Actuators, A 20, 135 (1989).

4. A. Karimi, O.R. Shojaei, T. Kruml, and J.L. Martin: Characterisation of tin thin films using the bulge test and the nanoindentation technique. Thin Solid Films 308-309, 334 (1997).

5. Y. Xiang, X. Chen, and J.J. Vlassak: Plain-strain bulge test for thin films. J. Mater. Res. 20, 2360 (2005).

6. G. Binnig, C.F. Quante, and Ch. Gerber: Atomic force microscope. Phys. Rev. Lett. 56, 930 (1986).

7. J.J. Vlassak and E.D. Nix: A new bulge testing technique for the determination of Young's modulus and Poisson's ratio of thin films. J. Mater. Res. 7, 3242 (1992).

8. E.I. Bromley, J.N. Randall, D.C. Flanders, and R.W. Mountain: A technique for the determination of stress in thin films. J. Vac. Sci. Technol., B 1, 1364 (1993).

9. A.J. Kalman, A.H. Verbruggen, and G.C.A.M. Jannsen: A novel bulge-testing seup for rectangular free-standing thin films. Rev. Sci. Instrum. 70, 4026 (1999).

10. A. Catlin and W.P. Walker: Mechanical properties of thin singlecrystal gold films. J. Appl. Phys. 31, 2135 (1960).

11. M.K. Small and W.D. Nix: Analysis of the accuracy of the bulge test in determining the mechanical properties of thin films. J. Mater. Res. 7, 1553 (1992).

12. K. Durst, M. Göken, and H. Vehoff: Finite element study for nanoindentation measurements on two-phase materials. J. Mater. Res. 19, 85 (2004).

13. M. Göken, M. Kempf, M. Bordenet, and H. Vehoff: Nanomechanical characterization of metals and thin films. Surf. Interface Anal. 27, 302 (1999).

14. W.C. Oliver and G.M. Pharr: An improved technique for determining hardness and elastic modulus using load and displacement sensing indentation experiments. J. Mater. Res. 7, 1564 (1992).

15. Hütte-Das Ingenieurwissen, 32nd ed., edited by H. Czichos and M. Hennecke (Springer-Verlag, Heidelberg, 2004), p. E66.

16. Y-G. Jung, B.R. Lawn, M. Martyniuk, H. Huang, and X.Z. Hu: Evaluation of elastic modulus and hardness of thin films by nanoindentation. J. Mater. Res. 19, 3076 (2004).

17. A.R. Reinberg: Plasma deposition of inorganic thin films. Аnnu. Rev. Mater. Sci. 9, 341 (1979).

18. User manual: Dimension $3100^{\mathrm{TM}}$ Scanning Probe Microscope. Digital Instruments-Veeco Metrology Group, Chadds Ford, PA, 2003.

19. J.J. Vlassak: New experimental techniques and analysis methods for the study of mechanical properties of materials in small volumes. Ph.D. Dissertation, Stanford University, Stanford, CA, 1994.

\section{APPENDIX A: STRESS AND STRAIN IN LONG RECTANGULAR MEMBRANES ${ }^{19}$}

Imagine a cylindrical pipe bearing an internal pressure $p$ which is cut in two halves along its axis of symmetry [Fig. A1(a)]. The wall thickness, the pipe length, and the radius of curvature are represented by $t, l$, and $R$, respectively. By balancing the forces, one finds

$$
\begin{aligned}
2 R l p & =2 t l \sigma, \\
& \Rightarrow \sigma=\frac{R p}{t},
\end{aligned}
$$

where $\sigma$ is the stress in circumferential direction. Simple geometric relations show that, for $h \ll a$ :

$$
\begin{aligned}
R^{2} & =(R-h)^{2}+a^{2} \\
& =R^{2}-2 R h+h^{2}+a^{2} \\
& \Rightarrow R=\frac{h^{2}+a^{2}}{2 h} \approx \frac{a^{2}}{2 h} .
\end{aligned}
$$

Inserting Eq. (A2) in Eq. (A1) yields

$$
\sigma_{x}=\frac{p a^{2}}{2 t h}
$$

The strain is obtained purely from geometric considerations as well [Fig. A1(b)]:

$$
\epsilon=\frac{R \theta-a}{a}=\frac{\theta-(a / R)}{a / R} .
$$

The Taylor expansion of $\theta$ is

$$
\theta=\arcsin (a / R)=(a / R)+\frac{(a / R)^{3}}{6}+\cdots
$$




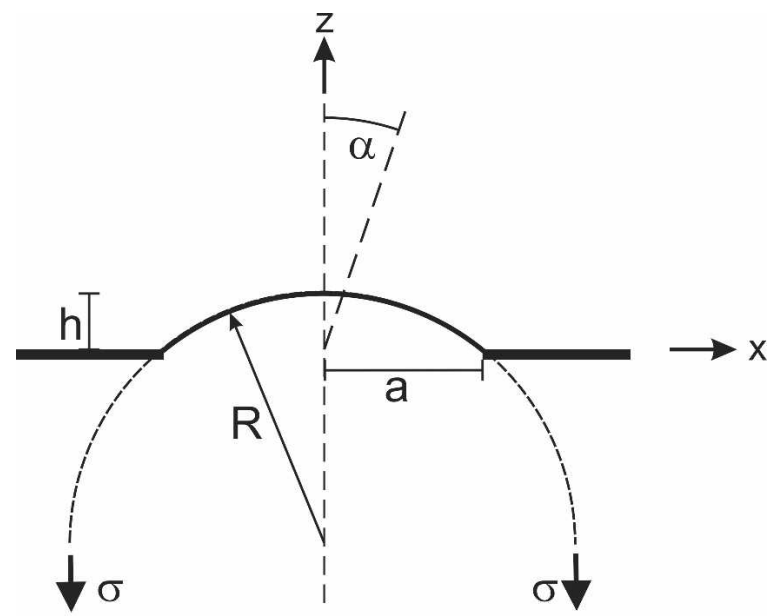

(a)

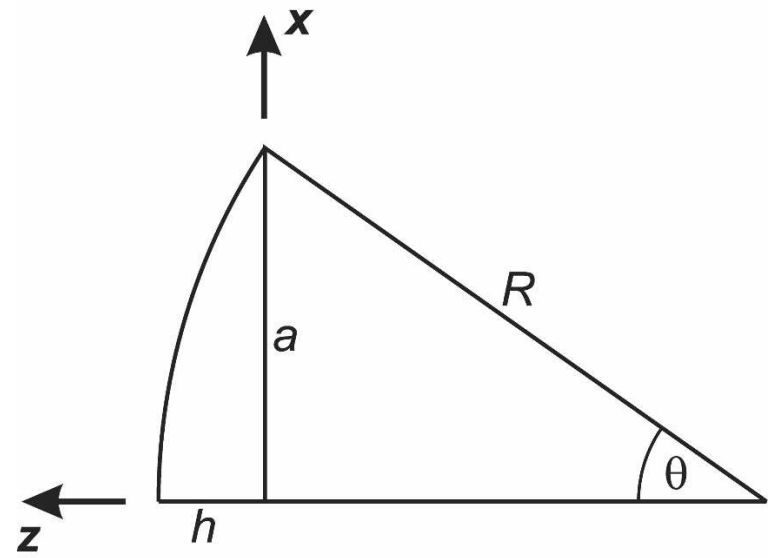

(b)

FIG. A1. (a) Equilibrium of the wall forces and the force resulting from the internal pressure; effect of angular misalignment $\alpha$ on the displacement detection. (b) Derivation of the film strain $\epsilon_{x}$ from geometrical parameters.

Therefore

$$
\epsilon=\frac{(a / R)+\frac{(a / R)^{3}}{6}-(a / R)}{(a / R)}=\frac{a^{2}}{6 R^{2}} .
$$

Substituting $R$ by Eq. (A2) yields

$$
\epsilon=\frac{2 h^{2}}{3 a^{2}} .
$$

\section{APPENDIX B: DISPLACEMENT DETECTOR ALIGNMENT}

During system operation the laser detector can be misaligned in two ways: lateral misalignment in the $x$ direction and angular misalignment by tilting the stage relative to the laser axis.

\section{Lateral misalignment}

According to the bulge model, the membrane shape in the $x-z$ plane is described as a segment of a circle. To have the origin of the coordinate system where it previously has been defined, the circular equation reads

$$
x^{2}+(z+R+h)^{2}=R^{2},
$$

where $R$ is the radius of the circle. Using Pythagoras' theorem, one finds

$$
R=\frac{h^{2}+a^{2}}{2 h}
$$

insertion in Eq. (A1) yields

$$
z(x, a, h)=\frac{1}{2 h}\left(h^{2}-a^{2}+\sqrt{h^{4}+2 h^{2} a^{2}+a^{4}-4 x^{2} h^{2}}\right),
$$

for all $z>0$. Negative values of $z$ are not relevant in this consideration.

To check the lateral alignment accuracy of the setup, the light microscope of the AFM was focused on a prominent surface feature, and its coordinates relative to the origin of the AFM stage were recorded. Afterward, the stage was first moved to an arbitrary position and then repositioned to the defined coordinates. The effective distance between this point and the position of the prominent feature was determined to $\Delta x=70.7 \mu \mathrm{m}$ and $\Delta y=59.0 \mu \mathrm{m}, \Delta x$ and $\Delta y$ being the average misalignment in the $x$ and $y$ directions, respectively. If the alignment process is made strictly unidirectional, thus eliminating the backslash of threads, these values decrease to $39.7 \mu \mathrm{m}$ and $27.0 \mu \mathrm{m}$, respectively. Assuming a membrane half width of $a=1 \mathrm{~mm}$ and a membrane displacement of $h=70 \mu \mathrm{m}$, which are reasonable values, Eq. (A3) yields a relative error in displacement detection of $0.157 \%$ and $0.0084 \%$ for $\Delta x=39.7 \mu \mathrm{m}$ and $\Delta y=29.0 \mu \mathrm{m}$, respectively.

\section{Angular misalignment}

To study the effect of sample tilting, the circle segment model is extended by a straight line representing the laser beam which passes the origin and is tilted by $\alpha$. We assume perfect lateral alignment of the laser beam [Fig. A1(a)]. Hence, the trajectory of the laser beam is described by the simple equation

$$
l(x)=\tan \left(90^{\circ}-\alpha\right) x=\cot (\alpha) x .
$$

The $x$ coordinate of the point of intersection is 


$$
\begin{gathered}
x_{\mathrm{int}}=\frac{1}{2 h(\tan (\alpha)+1)}\left[h^{2}-a^{2}+A \tan (\alpha)\right], \\
A=\sqrt{h^{4}+2 h^{2} a^{2}+a^{4}+4 h^{2} a^{2} \tan ^{2}(\alpha)} .
\end{gathered}
$$

The corresponding $y$ coordinate is

$$
y_{\text {int }}=x_{\text {int }} \cot (\alpha) \text {. }
$$

The measured displacement is the distance between the origin and the point of intersection

$$
h_{\text {meas }}=\sqrt{x_{\text {int }}^{2}+y_{\text {int }}^{2}} .
$$

Therefore, the error in the measurement equals

$$
\begin{aligned}
\Delta h= & \frac{h_{\text {meas }}-h}{h} \\
= & \frac{1}{2 h\left(\tan ^{2}(\alpha)+1\right)} \sqrt{\left(h^{2}-a^{2}+A\right)^{2} \tan ^{2}(\alpha)\left(\cot ^{2}(\alpha)+1\right)} \\
& -1 .
\end{aligned}
$$

To stay below a detection error of $1 \%$, a misalignment from the normal direction of $8^{\circ}$ is tolerable, assuming the same values for $a$ and $h$ as mentioned above. This criterion is easily met in this experimental setup. 